\title{
EFECTOS SOBRE LA CUBIERTA VEGETAL, LA ESCORRENTÍA Y LA EROSIÓN DEL SUELO, DE LA ALTERNANCIA CULTIVO- ABANDONO EN PARCELAS EXPERIMENTALES
}

\author{
Belmonte Serrato, F.; Romero Díaz, A.; López Bermúdez, F. ${ }^{1}$
}

\section{RESUMEN}

Se presentan resultados de las consecuencias hidrológicas y erosivas derivadas de la alternancia cultivo-abandono, obtenidos en parcelas de erosión del campo experimental de «El Ardal» (Murcia, Sureste de España). Los primeros resultados, parecen indicar que en esta área, el abandono de campos de cultivo, permite un aumento de cobertura vegetal, herbáceas anuales en la primera fase, que van siendo sustituidas posteriormente por especies perennes del matorral circundante (Sideritis leucantha, Teucrium capitatum, helichrysum stoechas). Este aumento de la cobertura vegetal unido a un aumento de la compactación del suelo, ocasiona un incremento del porcentaje de escorrentía, y a la vez, una disminución de la carga de sedimentos, que se traduce en una menor erosión. La intensidad de la lluvia se revela, una vez más, como uno de los parámetros fundamentales en la generación de escorrentía y pérdida de suelo.

Palabras clave: Parcelas de erosión, cultivo, abandono, escorrentía, sedimentos, medio semiárido.

\section{SUMMARY}

Are presented results of the hydrological consequences and soil erosion derivatives from the alternations cultivate-abandonment, obtained in erosion plots of the experimental field from The Ardal (Murcia, South-East of Spain). The first results, seem to indicate that in this area, with its special environmental characteristic, the abandonment of cultivation fields, permits an increase in vegetable coverage, grassy annual in the first phase, that go being substituted thereinafter by perennial kinds of the surrounding scrub (Sideritis leucantha, Teucrium capitatum, helichrysum stoechas). This increase in the vegetable coverage united to an increase in crushing of the soil, causes an increase in the percentage of runoff, but at the same time, a decrease with the sediments load, that is translated in a smaller erosion. The intensity of the rain is shown, once again, as one of the fundamental parameters in the generation of runoff and loss of soil.

Key words: Erosion plots, cultivation, abandonment, runoff, sediments, semiarid environment.

1 Los tres autores, son profesores de la Universidad de Murcia. 


\section{Introducción}

Es sabido que la erosión de los suelos está ligada a factores naturales (clima, litología, pendiente, vegetación, etc.), sin embargo, el hombre puede modificar muchas de las variables que intervienen en los procesos de erosión agudizándolos en unos casos, o incluso creándolos en otros (Romero Díaz, et al., 1998).

La fragilidad de los medios semiáridos, hace muy difícil la recuperación de las áreas sometidas a cultivo cuando estas son abandonadas (García Ruiz et al., 1996). Las dificultades de la vegetación para recolonizar las áreas abandonadas, condiciona que estas queden a merced de los procesos de erosión que empiezan a actuar rápidamente favorecidos por la frecuente torrencialidad de las precipitaciones mediterráneas. En general, el abandono de tierras de cultivo suele ir acompañado de un aumento de los procesos de erosión y degradación del suelo, que frecuentemente acaba con un acarcavamiento generalizado de las laderas anteriormente cultivadas, lo que hace todavía más difícil la recuperación de la vegetación natural (Ruiz Flaño, 1993).

Sin embargo, en algunas ocasiones, las características climáticas y edáficas de algunas áreas, pueden favorecer tras el abandono, la recuperación más o menos rápida de la cobertura vegetal, invirtiendo la dinámica de los procesos de erosión y permitiendo a medio plazo la recuperación de la vegetación natural. En este sentido, Padilla Blanco (1998), lleva a cabo un trabajo riguroso y en profundidad de la colonización vegetal en campos abandonados en al provincia de Alicante. Otros autores, coinciden también en señalar, que en ausencia de litologias blandas y de episodios lluviosos intensos tras el abandono, la vegetación coloniza rápidadmente los campos abandonados, reduciendo, en consecuencia, el riesgo de erosión (Marco Molina y Morales Gil, 1995; Molina Gallart y Nadal Tersa, 1998; Camarasa Belmote et al., 1998).

Una de las líneas maestras de investigación que se está llevando acabo en el área experimental de «El Ardal», es la de evaluar en parcelas de erosión, los cambios hidrogeomorfológicos y biofísicos que se producen, fundamentalmente en generación de escorrentía y producción de sedimentos, en una alternancia cultivo-abandono.

\section{Área de estudio}

El estudio se ha realizado en el campo experimental de «El Ardal» situado en la Cuenca del Río Mula (Murcia, Sureste de España) (Figura 1). Aquí, el Área de Geografía Física de la Universidad de Murcia, viene realizando desde 1989, un seguimiento y evaluación de los procesos de erosión y de las relaciones clima-planta-suelo (Martínez Fernández et al., 1995; ICONA, 1996; López-Bermúdez et al., 1998).

El campo está situado a unos $550 \mathrm{~m}$ de altitud, en una pequeña loma con orientación Norte. La precipitación media anual está en torno a los $300 \mathrm{~mm}$, aunque con acusadas variaciones mensuales e interanuales (Belmonte Serrato y Romero Díaz, 1996), siendo la temperatura media de $14.5^{\circ} \mathrm{C}$; pertenece al piso bioclimático Mesomediterráneo y al ombrotipo semiárido.

El Ardal se encuadra en la provincia biogeográfica Castellano-Maestrazgo-Manchega, sector Manchego, subsector Manchego-Espunense (Sánchez Gómez et al., 1998). La formación vegetal predominante es el matorral, compuesto mayoritariamente por arbustos y gramíneas perennes, siendo Rosmarinus officinalis y Brachypodium retusum, las especies más representativas respectivamente, a estas se suman otras como Juniperus 
oxycedrus, Rhamnus lycioides, Thymus vulgaris, Thymus membranaceus y de forma aislada Pinus halepensis. En general, el porcentaje de cobertura es bastante alto, oscila en torno al $60 \%$.

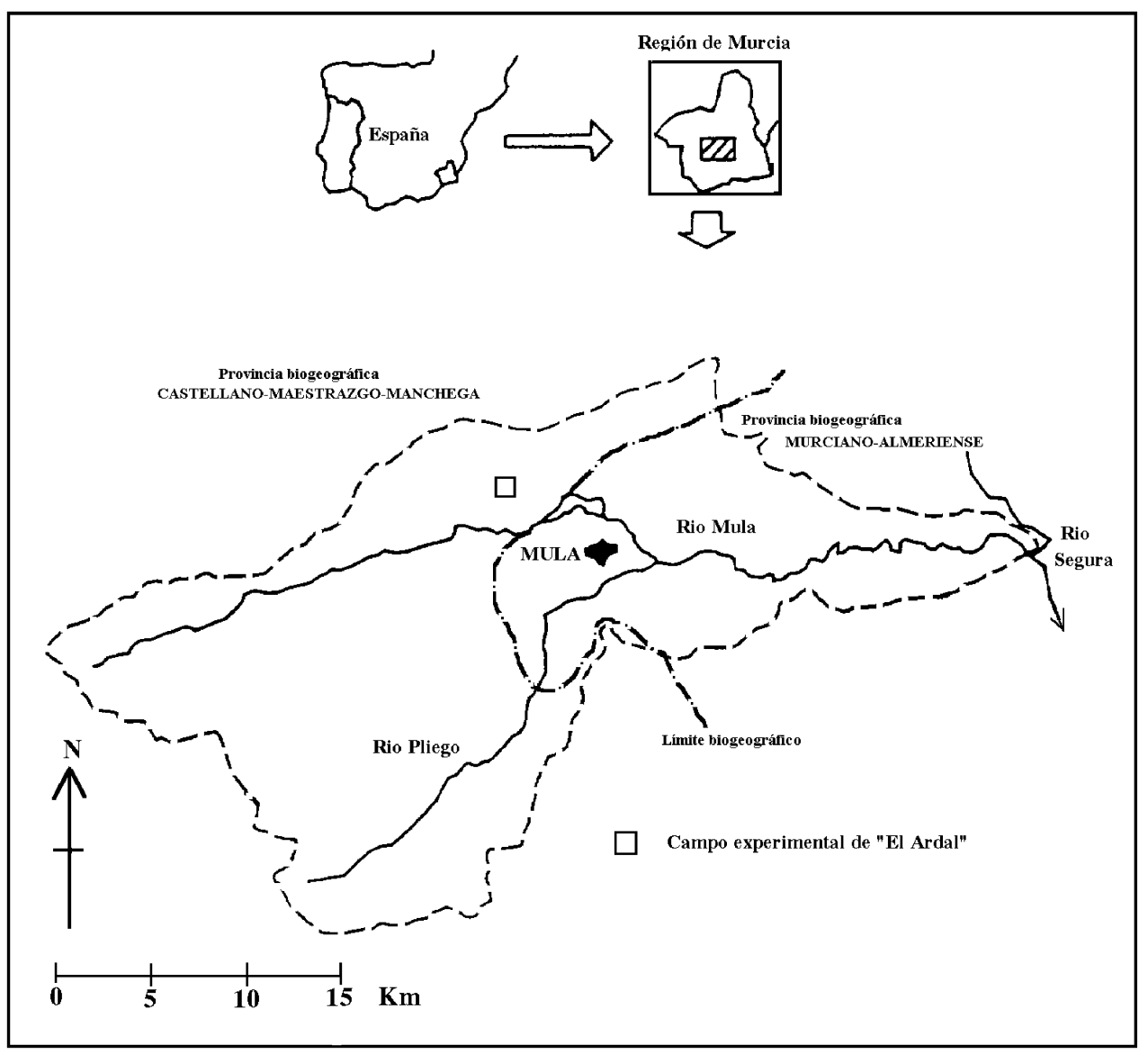

FIGURA 1. Localización del área de estudio.

Los suelos predominantes son calcisoles pétricos, poco profundos y pedregosos, que se extienden sobre calizas o conglomerados calizos en las partes más altas de la ladera, o sobre un horizonte petrocálcico, a veces muy cementado, siendo por ello suelos con un alto contenido en carbonato cálcico (más del 50\%). La textura es limo-arcillosa en las partes altas de la ladera y arenosa en el sector cultivado. El contenido de materia orgánica es elevado en el matorral (5-7\%) pero muy bajo en los suelos cultivados (1\%). La humedad del suelo es muy baja y la permeabilidad en general muy elevada; siendo de destacar la notable reducción de la permeabilidad en los sectores de suelo desnudo en comparación con las áreas cubiertas de matorral (Martínez Fernández et al. 1995). 


\section{Metodología}

\section{a) Instrumentación y obtención de datos}

Los parámetros climáticos se han obtenido de la estación meteorológica automática con que está dotado el Campo experimental de «El Ardal» y para la precipitación, además de los datos de la estación, los registrados en dos pluviómetros totalizadores instalados en las parcelas.

El control de la escorrentía y las pérdidas de suelo, se lleva a cabo en un grupo de seis parcelas, con dimensiones de $2 \times 8 \mathrm{~m}$, pendiente del $7 \%$ y orientación $\mathrm{N}$. Para la recogida de la escorrentía y los sedimentos, se colocó un depósito de 200 litros, y dentro de él, un recipiente de 12 litros que es suficiente para recoger la escorrentía del $90 \%$ de los episodios que se producen en esta zona. El agua y los sedimentos se miden y con posterioridad tras homogeneizar la mezcla, se extrae una muestra de 0.5 litros, que se introduce en la estufa hasta la completa evaporación del agua, se pesan los sedimentos y se obtiene así la cantidad total de sedimentos. Con estos datos se calculan las tasas de erosión y escorrentía de cada evento lluvioso.

Las cuatro primeras parcelas fueron instaladas en 1989 en un campo de cultivo en el extremo superior limítrofe con el matorral contiguo y en 1990 se instalan otras dos, por lo que el período considerado se inicia en este último año. A las parcelas se les aplicó distintos tipos de prácticas agrícolas, similares a las prácticas de cultivo del área circundante (tabla 1). De 1990 a 1994, ambos inclusive, se mantuvo el tratamiento agrícola y en 1995, año muy seco, se inició el período de abandono, con el objetivo de evaluar las consecuencias de la retirada de las prácticas agrícolas.

Tabla 1

PARCELAS, TIPOS DE PRÁCTICA DE CULTIVO Y USOS

\begin{tabular}{|c|l|c|c|}
\hline Parcela $\mathbf{N}^{\mathbf{0}}$ & Tipo de práctica de cultivo & Cultivo & Abandono \\
\hline 1 & Cereal (Cebada) & $90-94$ & $95-97$ \\
2 & Testigo (con piedras) & $90-94$ & $95-97$ \\
3 & Testigo (sin piedras) & $90-94$ & $95-97$ \\
4 & Arada con surcos en sentido pendiente & $90-94$ & $95-97$ \\
11 & Arada sin surcos & $90-94$ & $95-97$ \\
12 & Cereal (Trigo) & $90-94$ & $95-97$ \\
\hline
\end{tabular}

\section{b) Análisis de los datos}

La variabilidad interanual, en volumen e intensidad, dificulta un análisis comparativo directo, es decir, referido a datos absolutos, entre los dos períodos. Para poder comparar los dos períodos, se han obtenido promedios anuales, tanto de escorrentía como de sedimentos, de las dos parcelas de cereal y de las dos parcelas testigo, de modo que los tipos de prácticas son: cereal, testigo, arado con surcos y arado. Luego para cada práctica se obtuvieron los promedios de cada período de uso (período de cultivo y período de aban- 
dono). Finalmente para eliminar el efecto de la variabilidad de las precipitaciones en ambos períodos, se ponderaron los promedios de las prácticas de cereal, arado y arado con surcos, estandarizándolos con los promedios testigo en cada período, admitiendo que al no producirse cambio de uso en las parcelas testigo, las diferencias entre ambos períodos se deben a las diferencias en las características de los episodios de lluvia, y, por tanto, las diferencias entre períodos del resto de prácticas puestas en relación con las testigo, se deben al cambio de uso (cultivo-abandono).

\section{Resultados}

\section{a) Precipitación y cubierta vegetal}

Se ha de tener en cuenta que los dos períodos comparados son distintos en cuanto al número de años considerados, 5 para el período de cultivo y 3 para el de abandono. No obstante, la utilización de datos medios permite comparar ambos períodos. En la tabla 2, se resumen las características anuales de precipitación, intensidades, número de episodios con generación de escorrentía, episodios con intensidad máxima superior a $10 \mathrm{~mm} / \mathrm{h}$, que son aquellos que generan más del 95\% de los sedimentos anuales, si bien sólo suponen un $48 \%$ del total de episodios, $\mathrm{y}$ el factor $\mathrm{R}$ de erosividad pluvial basado en el índice de Wischmeier (EI30), siendo el valor medio anual EI30/1000 (Morgan, 1986).

La precipitación media anual es muy similar en ambos períodos, aunque ligeramente favorable en los años de abandono. Sin embargo, las diferencias se acentúan en el resto de factores considerados, de modo que puede decirse que en el período de abandono los episodios son más intensos y sobre todo hay mayor número de sucesos con intensidad máxima superior a $10 \mathrm{~mm} / \mathrm{h}$. Esto queda reflejado en el índice medio de erosividad pluvial en cada período, 37 en el período de cultivo y 66.6 en el de abandono.

Tabla 2

CARACTERÍSTICAS DE LAS PRECIPITACIONES ANUALES Y FACTOR R DE EROSIVIDAD PLUVIAL

\begin{tabular}{|c|c|c|c|c|c|c|c|}
\hline Práctica & Años & $\underset{(\mathbf{m m})}{\mathbf{P .}}$ & $\begin{array}{c}\text { I. media } \\
(\mathrm{mm} / \mathrm{h})\end{array}$ & $\begin{array}{l}\text { I. media } \\
\text { max. } \\
(\mathrm{mm} / \mathrm{h})\end{array}$ & $\begin{array}{l}\mathrm{N}^{0} \text { episod. } \\
\text { con } \\
\text { escorren. }\end{array}$ & $\begin{array}{c}\text { Epis. } \\
>10 \mathrm{~mm} / \mathrm{h} \\
\text { I.maxima }\end{array}$ & $\begin{array}{c}\text { Factor R } \\
\mathrm{J} / \mathrm{mm} / \mathrm{m}^{2} / \mathrm{h}\end{array}$ \\
\hline \multirow{5}{*}{ Cultivo } & 1990 & 384.5 & 6.9 & 18.9 & 15 & 5 & 70.8 \\
\hline & 1991 & 293.5 & 3.5 & 13.7 & 12 & 4 & 28 \\
\hline & 1992 & 307 & 2.7 & 11.6 & 9 & 4 & 31.6 \\
\hline & 1993 & 255 & 4.3 & 17.4 & 15 & 8 & 38.1 \\
\hline & 1994 & 156.5 & 5.8 & 19.1 & 8 & 6 & 16.4 \\
\hline \multirow{3}{*}{ Abandono } & 1995 & 117.6 & 6.4 & 18.8 & 7 & 4 & 18.4 \\
\hline & 1996 & 357.6 & 5.6 & 13.4 & 19 & 10 & 46.5 \\
\hline & 1997 & 423.4 & 7.2 & 30.3 & 14 & 7 & 134.9 \\
\hline Cultivo & med. & 279.3 & 4.7 & 16.4 & 10.4 & 5.2 & 37 \\
\hline Abandono & med. & 299.5 & 6.4 & 20.8 & 13.3 & 7 & 66.6 \\
\hline
\end{tabular}


La cubierta vegetal en el período de cultivo oscila en las parcelas de cereal, entre un $0 \%$ en Verano y Otoño a un $90 \%$ en Invierno y Primavera, en las parcelas aradas oscila entre el $0 \%$ y el $10 \%$, y en las testigo se sitúa en torno al $30 \%$.

En el período de abandono, pasa de $0 \%$ en las parcelas cultivadas en 1995, primer año de abandono (año además extremadamente seco), a un 60\% en las de cereal en 1996 y 95\% en 1997 (sin oscilaciones estacionales acusadas), y un 20\% en las aradas en 1996 y $50 \%$ en 1997. Esta diferencia de cobertura, entre las parcelas de cereal y las aradas (figuras 2 y 3), es debido a que durante la siembra se hacia un abonado en las parcelas de cereal y no en las aradas, de modo que la mayor presencia de nutrientes permitía, tras el abandono, un mayor aumento de cobertura vegetal, «malas hierbas», y plantas de cereal de las semillas caídas en la última cosecha. En las parcelas testigo, en 1995 la cobertura se reduce a un $30 \%$ aproximadamente, debido al escaso desarrollo de las especies anuales; en 1996 se recupera y llega a un $40 \%$ y a un $60 \%$ en 1997.

Respecto a la composición de especies colonizadoras, hay una gran diferencia entre las parcelas testigo y aquellas en las que se realizaban prácticas agrícolas. Mientras que en las primeras predominan especies perennes, fundamentalmente fanerófitos como: Rosmarinus officinalis, Artemisia campestris, Helichrysum stoechas, Helianthemun almeriense, Sideritis leucantha y caméfitos como: Thymus zygis, Teucrium capitatun, Fumana thymifolia), que indican una recuperación paulatina del matorral tras nueve años de abandono, en las parcelas donde se realizó tratamiento agrícola tras el abandono, aparecen las herbáceas, hemicritófitos y terófitos fundamentalmente, (Raspistrum rugosum (en las parcelas cultivadas antes con cereal), Pallenis spinosa, Eryngium campestre, Plantago albicans, Atractylis humilis). En 1997 ya empieza a ser apreciable la cobertura de especies más propias del matorral como: Artemisia campestris, Heliantemun almeriensis, Sideritis leucantha, y en la parcela 4 una plántula de Rosmarinus officinalis.

\section{b) Tasas anuales de escorrentía y sedimentos}

Tanto el coeficiente de escorrentía como la concentración y producción de sedimentos varían considerablemente entre unos años y otros (tabla 3). Pero en los tres años del período de abandono, se observa un espectacular aumento con respecto a los cinco años del período de cultivo. En este sentido, el año 1995 es muy significativo, con la precipitación más baja de todo el período y un rango de intensidades muy similar al resto, tiene los valores más elevados de concentración de sedimentos, que en el caso de la parcela 4, alcanza los 178 $\mathrm{g} / \mathrm{l}$. Estos valores pueden explicarse por la ausencia de cubierta vegetal que provoca el abandono, la escasa precipitación invernal, el largo período de sequía estival y la correlativa desagregación de las partículas del suelo. Esta respuesta también se registra en las parcelas testigo, que ven reducida su cobertura, como consecuencia de la escasa germinación de las herbáceas anuales.

En 1996, el gran aumento de cobertura vegetal, el mayor grado de compactación del suelo y la menor intensidad de los episodios de lluvia, devuelve la concentración de sedimentos casi a los valores «normales» del período de cultivo, pero en 1997 debido a la fuerte intensidad de las lluvias y aumento del coeficiente de escorrentía, vuelven a obtenerse valores elevados de concentración de sedimentos, a pesar del aumento de cobertura vegetal. 

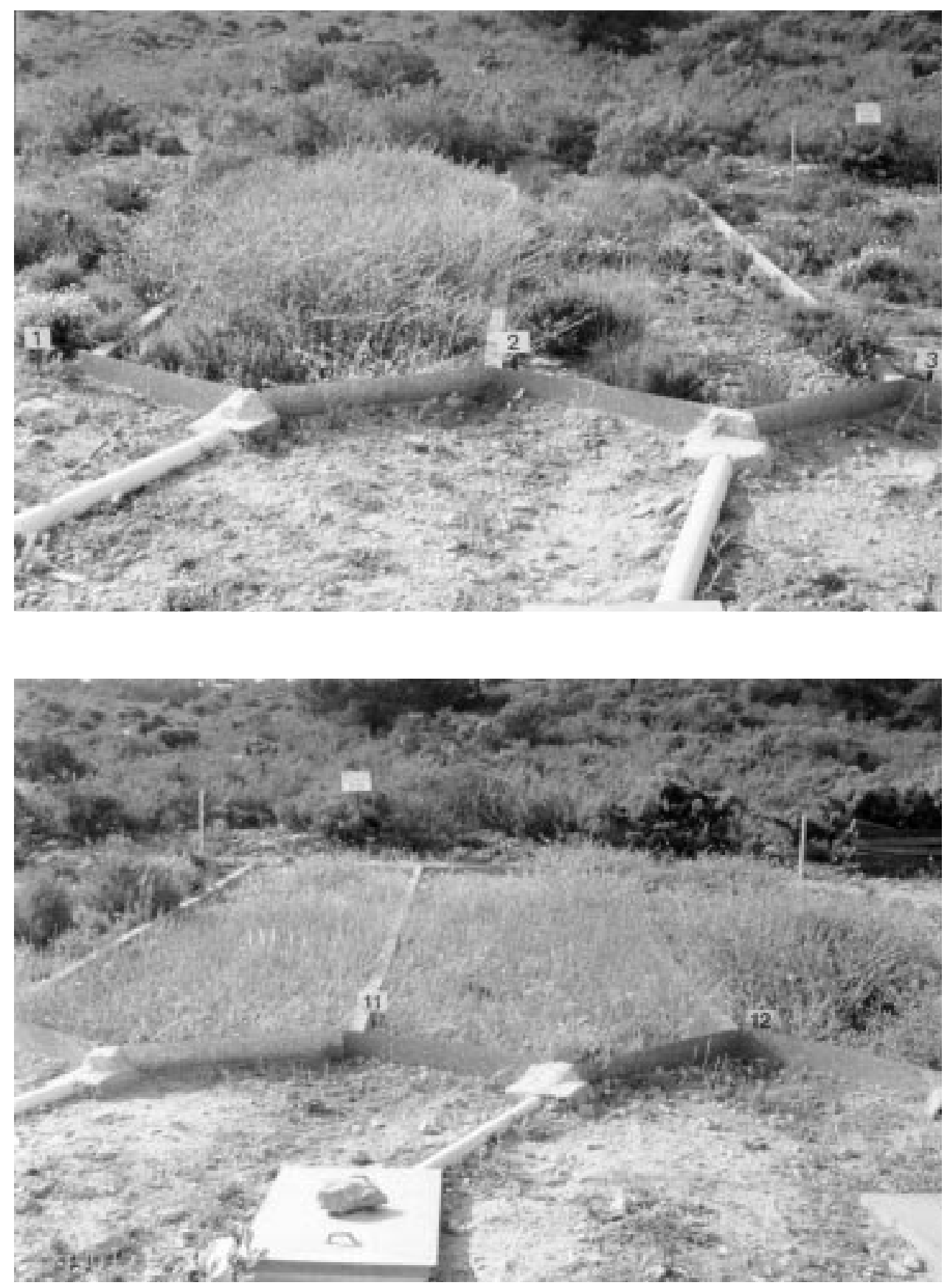

FiguRA 2 y 3. Vista parcial del Campo Experimental de El Ardal y del dispositivo de las parcelas de erosión con distintos usos del suelo. 
Tabla 3

VALORES ANUALES DE ESCORRENTÍA (\%), CONCENTRACIÓN (GR/L) Y PRODUCCIÓN DE SEDIMENTOS (G/M²)

\begin{tabular}{|l|l|r|r|r|r|r|r|r|r|}
\hline \multicolumn{2}{|c|}{} & $\mathbf{1 9 9 0}$ & $\mathbf{1 9 9 1}$ & $\mathbf{1 9 9 2}$ & $\mathbf{1 9 9 3}$ & $\mathbf{1 9 9 4}$ & $\mathbf{1 9 9 5}$ & $\mathbf{1 9 9 6}$ & $\mathbf{1 9 9 7}$ \\
\hline \multirow{3}{*}{ Testigo } & $\%$ & 1.4 & 4.4 & 1.3 & 1.0 & 1.7 & 2.0 & 2.1 & 7.7 \\
& $\mathrm{~g} / 1$ & 0.7 & 0.07 & 0.2 & 4.7 & 2.5 & 15.2 & 3.0 & 7.3 \\
& $\mathrm{~g} / \mathrm{m}^{2}$ & 3.9 & 0.9 & 0.9 & 12.1 & 6.5 & 34.9 & 22.0 & 238.3 \\
\hline \multirow{4}{*}{ Cereal } & $\%$ & 2.9 & 3.4 & 0.9 & 2.0 & 3.5 & 2.9 & 4.9 & 9.3 \\
& $\mathrm{~g} / 1$ & 2.9 & 0.6 & 0.4 & 3.2 & 14.1 & 42.4 & 13.9 & 6.3 \\
& $\mathrm{~g} / \mathrm{m}^{2}$ & 32.1 & 6.1 & 1.1 & 38.6 & 77.1 & 144.1 & 235.9 & 247.2 \\
\hline \multirow{3}{*}{ Surcos } & $\%$ & 0.6 & 5.7 & 2.4 & 5.7 & 7.5 & 8.4 & 9.1 & 13.6 \\
& $\mathrm{~g} / 1$ & 1.4 & 3.7 & 0.9 & 12.6 & 18.6 & 178.1 & 29.5 & 34.1 \\
& $\mathrm{~g} / \mathrm{m}^{2}$ & 3.1 & 62.5 & 6.8 & 184.1 & 217.4 & 1762.9 & 954.5 & 1948 \\
\hline \multirow{3}{*}{ Arada } & $\%$ & 2.0 & 5.4 & 2.4 & 1.9 & 1.7 & 5.3 & 6.0 & 12.5 \\
& $\mathrm{~g} / 1$ & 1.9 & 0.1 & 0.2 & 7.1 & 9.1 & 30.4 & 10.0 & 10.5 \\
& $\mathrm{~g} / \mathrm{m}^{2}$ & 14.5 & 1.6 & 1.8 & 34.8 & 25.2 & 191.2 & 295.0 & 356.2 \\
\hline
\end{tabular}

En 1995 y, sobre todo en 1997, se producen los chubascos más intensos de los ocho años. En los tres años del período de abandono, se producen 7 de los 10 episodios de intensidad media superior a los $10 \mathrm{~mm} / \mathrm{h}$. En concreto, el episodio ocurrido el 29 de septiembre de 1997, alcanza $100 \mathrm{~mm} / \mathrm{h}$ de intensidad máxima y $20 \mathrm{~mm} / \mathrm{h}$ de intensidad media, y genera en torno al $20 \%$ del total de sedimentos producidos en los ocho años. En la parcela arada con surcos en sentido de la pendiente, con dos años de abandono, estas últimas lluvias ocasionan una pérdida de suelo equivalente a $10 \mathrm{Tn} / \mathrm{ha}$. En consecuencia, una vez más se pone de manifiesto la importancia de la intensidad de la lluvia en relación con la producción de escorrentía y sedimentos (Romero Díaz et al., 1995).

\section{c) Promedios de escorrentía y sedimentos}

El aumento de intensidad de los episodios en el período de abandono provoca, como se ha constatado, un considerable aumento con respecto a los años del período de cultivo, tanto del coeficiente medio de escorrentía, como de la concentración de sedimentos y producción total de sedimentos. El coeficiente medio de escorrentía se multiplica por dos en las parcelas testigo, cereal y arada con surcos, y casi por tres en la parcela arada sin surcos. La concentración de sedimentos, es diez veces superior en las testigo y en la arada con surcos; ocho veces superior en la arada sin surcos; y más de 4 veces superior en las de cereal. La producción media se multiplica por más de 20 en las parcelas testigo; por 16 en la arada con surcos; por 18 en la arada sin surcos y por 7 en las de cereal (tabla 4).

Analizando únicamente los promedios de los valores absolutos, puede decirse que tras el abandono de las prácticas de cultivo se ha producido un aumento del coeficiente de escorrentía y sobre todo de la tasa de erosión, que pasa de una media de $0.37 \mathrm{Tn} / \mathrm{ha} / \mathrm{año}$, en el período de cultivo, a $6.8 \mathrm{Tn} / \mathrm{ha} / \mathrm{año}$ en el de abandono. Sin embargo, ya se ha mencionado que en 1996 y sobre todo en 1997, se produce un gran aumento de la cobertura vegetal, no 
sólo en las parcelas anteriormente cultivadas de cereal, sino también en las parcelas aradas, lo que debería haber permitido una mayor protección del suelo y por tanto, menores tasas de erosión, algo que en promedios absolutos no ha sucedido, debido a las características de las lluvias en este período.

Tabla 4

PROMEDIOS DE PRODUCCIÓN Y CONCENTRACIÓN DE SEDIMENTOS, Y

COEFICIENTE DE ESCORRENTÍA DE LOS PERÍODOS DE CULTIVO Y ABANDONO PARA LOS CUATRO TIPOS DE PRÁCTICA

\begin{tabular}{|l|ccc|ccc|}
\hline Uso & \multicolumn{3}{|c|}{ Cultivo } & \multicolumn{3}{c|}{ Abandono } \\
\hline Práctica & Esc. (\%) & Conc. (g/l) & Sed. (g/m $\left.\mathbf{m}^{2}\right)$ & Esc. $(\%)$ & Conc. $(\mathbf{g} / \mathbf{l})$ & Sed. $\left(\mathbf{g} / \mathbf{m}^{2}\right)$ \\
\hline Testigo & 1.9 & 0.8 & 4.8 & 3.9 & 8.5 & 98.4 \\
Cereal & 2.5 & 4.5 & 31.0 & 5.7 & 20.8 & 209.1 \\
A. surcos & 4.4 & 8.9 & 94.8 & 10.4 & 80.5 & 1555.1 \\
Arada & 2.7 & 2.0 & 15.6 & 7.9 & 16.9 & 280.8 \\
\hline
\end{tabular}

Esc $=$ Escorrentía $;$ Conc $=$ Concentración de sedimentos; Sed $=$ Sedimentos

\section{d) Estandarización de los promedios en relación a las parcelas testigo}

Para poder comparar ambos períodos minimizando los efectos del aumento de episodios intensos durante el período de abandono, se han estandarizado los promedios del coeficiente de escorrentía, concentración de sedimentos y producción de sedimentos de cada período, en relación a los obtenidos en las parcelas testigo, es decir, se han tomado los promedios de las testigo como unidad.

En este caso los resultados cambian con respecto a los obtenidos con los promedios absolutos. Para el coeficiente de escorrentía (figura 4), el abandono de las prácticas de cultivo, provoca un aumento del $12 \%$ en las parcelas de cereal, $17 \%$ en la arada con surcos y $47 \%$ en la arada sin surcos. Incrementos que se deben más al aumento de compactación superficial del suelo, que al de cobertura vegetal.

Sin embargo, los promedios estandarizados de concentración de sedimentos en gramos/litro, se invierten respecto a los promedios absolutos (figura 5). En este caso, se obtiene una menor concentración de sedimentos en los años de abandono de las prácticas agrícolas, el promedio de las parcelas de cereal se reduce en un 57\% respecto del promedio en el período de cultivo; en la parcela con surcos, la reducción es del 13\%; y en la parcela arada sin surcos, del $18 \%$.

Este resultado está mucho más acorde con lo esperado, ya que cuando las parcelas se encuentran aradas, es mucho más fácil la remoción y el arrastre de sedimentos que hace aumentar la concentración, ya que la lluvia no tiene que vencer ningún tipo de compactación superficial. La gran disminución que se ha producido en las parcelas de cereal, está en relación con el elevado porcentaje de cobertura vegetal, que reduce considerablemente la energía cinética de las gotas de lluvia, fundamentalmente en los meses de Verano y Otoño, que es cuando se producen las precipitaciones más intensas y cuando las parcelas durante el período de cultivo están desprovistas de vegetación. 


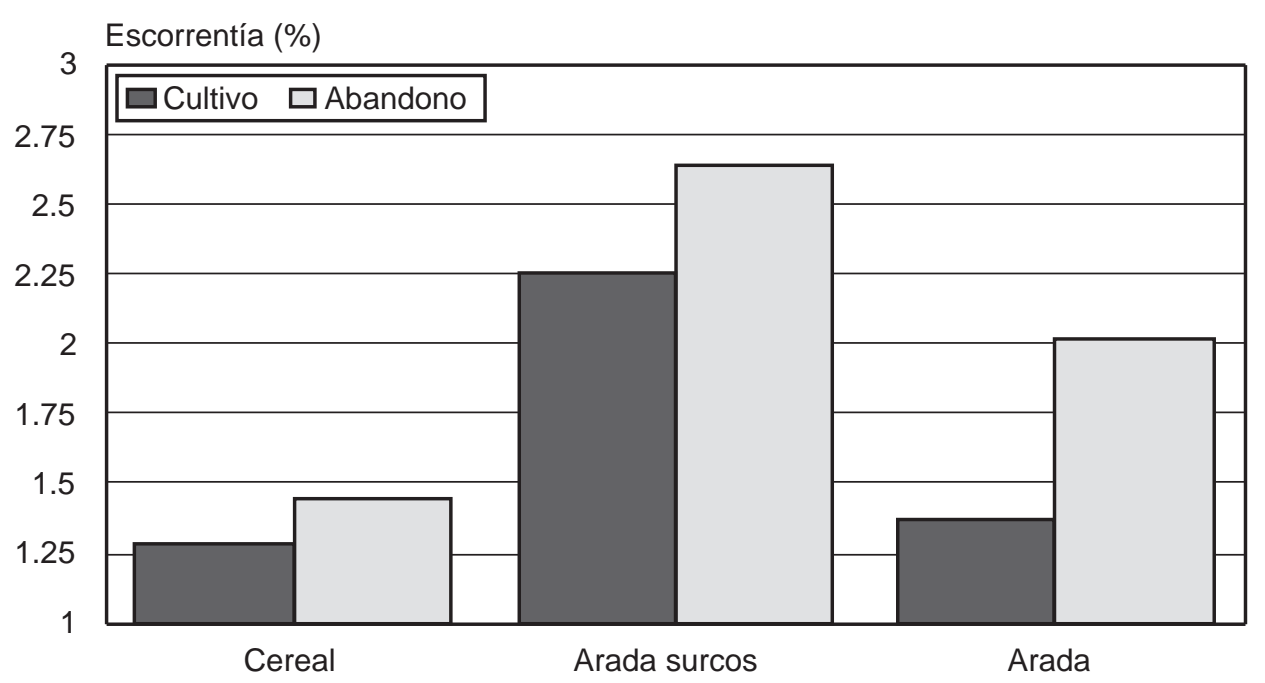

FIGURA 4. Coeficiente medio de escorrentía estandarizado al coeficiente medio de las parcelas testigo (testigo = 1).

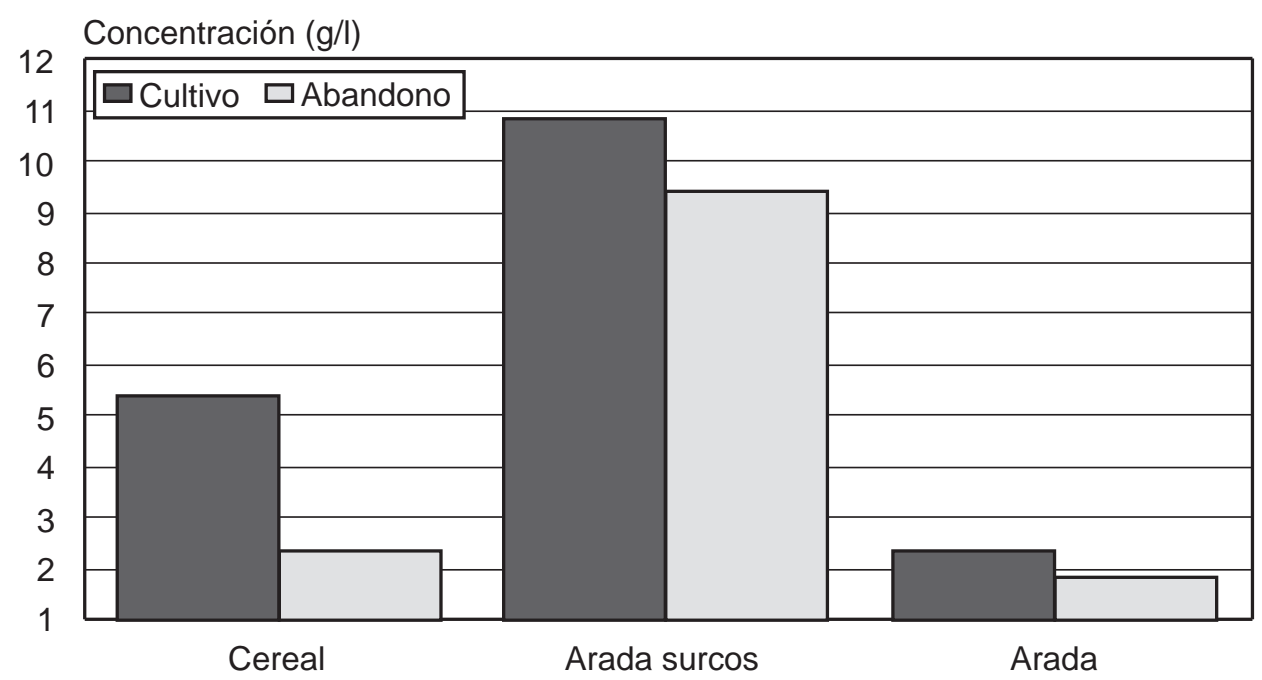

FIGURA 5. Concentración media de sedimentos estandarizada a la concentración media de las parcelas testigo (testigo $=1$ ). 
La disminución en la carga de sedimentos por litro de escorrentía, conduce, a pesar del aumento en el porcentaje de escorrentía, a una disminución de la producción de sedimentos, es decir de la erosión (figura 6). La tasa de erosión se reduce en un $67 \%$ en las parcelas de cereal, un $19 \%$ en la arada con surcos y un $11.5 \%$ en la arada sin surcos. La explicación de la notable reducción en la pérdida de suelo en las parcelas de cereal, es la misma que la que se daba en el caso de la concentración de sedimentos. Los menores porcentajes de cobertura vegetal en las parcelas aradas, facilitan que la energía cinética de la lluvia sea más eficaz que en las de cereal, en las que la cobertura llegaba al $95 \%$ en 1997.

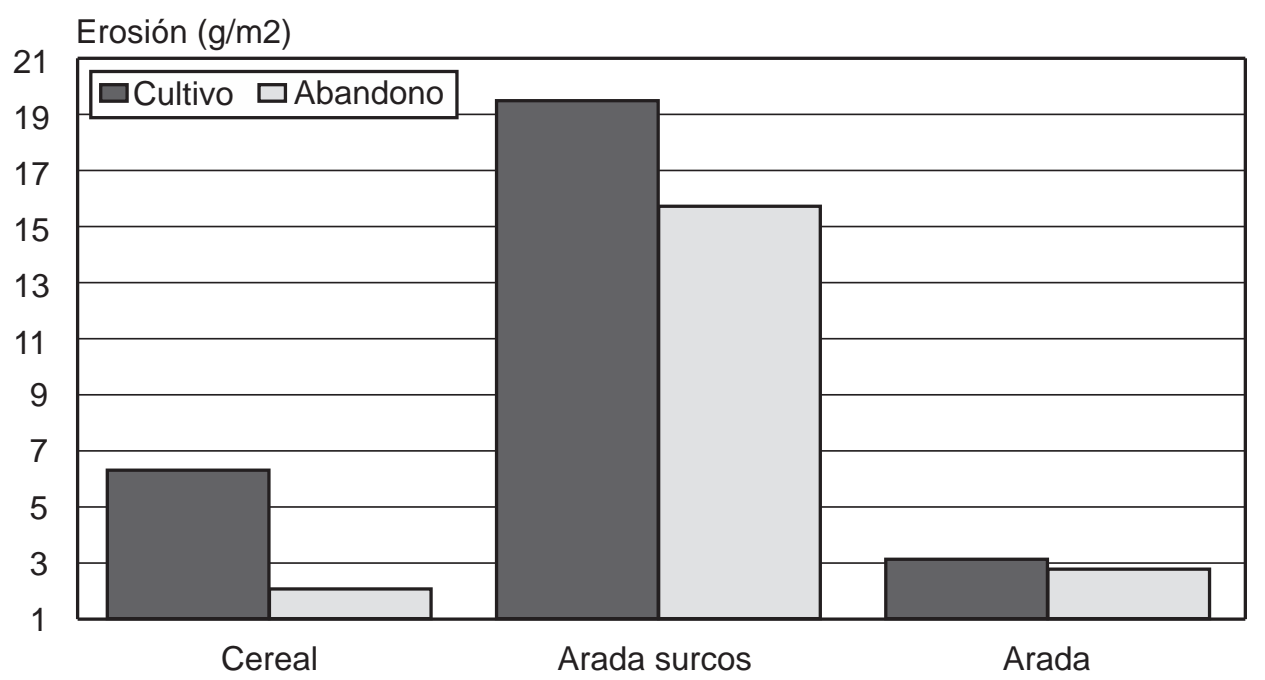

FiguRa 6. Producción media de sedimentos estandarizada a la producción media de las parcelas testigo (testigo $=1$ ).

\section{Discusión y conclusiones}

En el período de abandono, la precipitación media anual es un $7 \%$ superior a la del período de cultivo. Sin embargo, las intensidades media y media máxima son un 26 y un $21 \%$ superiores respectivamente, así como el número medio de episodios con escorrentía ( $22 \%$ superior) y el número medio de episodios con intensidad mayor de $10 \mathrm{~mm} / \mathrm{h}$ (un $26 \%$ superior en el período de abandono). Estas características definen la erosividad pluvial que es casi un $50 \%$ superior en el período de abandono.

En los tres años del período de abandono se produce un gran aumento en la cobertura vegetal de las parcelas. Las de cereal pasan de un $10 \%$ en 1995 a un $95 \%$ en 1997, las aradas pasan de $0 \%$ en 1995 al 50\% en 1997; y las testigo pasan del 20 al $60 \%$.

La composición de especies de las parcelas testigo, tras nueve años de abandono, indican una recuperación importante de especies propias del matorral circundante, que van desplazando a las herbáceas iniciales y otras especies de campos abandonados. En las parcelas de cereal y en las aradas se registra una cobertura mayoritaria de herbáceas y especies pro- 
pias de campos abandonados, así como una incipiente colonización de algunas especies pioneras del matorral. Este hecho ha sido observado en otros muchos lugares del Sureste peninsular (Marco Molina y Morales Gil, 1995; Padilla Blanco, 1998).

En 1995, primer año de abandono, la escasa precipitación $(117 \mathrm{~mm})$ no permitió el establecimiento de una cobertura vegetal que protegiese el suelo, además la elevada intensidad de los chubascos otoñales, tras la fuerte y dilatada sequía estival, ocasionó un fuerte aumento de producción de sedimentos, las concentraciones más elevadas de la serie. En 1996, se vuelve a coeficientes de escorrentía y concentraciones de sedimentos afines a los valores de los años de cultivo, pero en 1997, el gran volumen y la mayor intensidad de los episodios vuelve a ocasionar un elevado coeficiente de escorrentía y un aumento en la concentración de sedimentos, a pesar del considerable aumento de la cobertura vegetal y compactación del suelo.

Estas diferencias de volumen e intensidad de los acontecimientos lluviosos entre los dos períodos, reflejadas en unos índices de erosividad pluvial superiores, ocasionan promedios de escorrentía y concentración de sedimentos claramente favorables al período de abandono. El promedio de producción total de sedimentos multiplica por 15 el obtenido en los 5 años del período de cultivo.

$\mathrm{Al}$ relacionar los promedios de escorrentía y sedimentos de las parcelas cultivadas, con los promedios de las parcelas testigo, se observa que el coeficiente medio de escorrentía se eleva en el período de abandono, entre un 12 y un $47 \%$, con respecto al período de cultivo. Pero, la concentración de sedimentos se ve reducida entre un 13 y un $57 \%$ y la tasa de erosión se reduce entre un 11,5 y un $67 \%$, en relación al período de cultivo.

Con estos resultados puede concluirse que en el área de estudio, el abandono de las prácticas de cultivo en las parcelas, ha incrementado el coeficiente medio de escorrentía debido al aumento de compactación del suelo, pero ha favorecido la reducción de la tasa media de erosión, a causa del aumento de cobertura vegetal que hace disminuir la capacidad erosiva de la lluvia. No obstante, hay que advertir que, por las características biofísicas de esta área, especialmente por su litología caliza y cobertura vegetal, los resultados no pueden ser extendidos a otras áreas con características ambientales distintas y más frágiles (cuencas neogeno-cuaternarias de Mula o Fortuna), en donde el abandono de los cultivos, puede tener consecuencias más preocupantes para la conservación del recurso suelo.

\section{Agradecimientos}

Este trabajo se ha realizado en el marco de los Proyectos de Investigación MEDALUS (Mediterranean Desertification and Land Use), contrato N ${ }^{\circ}$ ENV4-CT95-0119 (DG-XIIDTEE), financiado por la Unión Europea (1991-1998); del AGF95-0635, financiado por la CICYT en el marco del Plan Nacional de I+D (1995-1998) y RESEL-LUCDEME (Dirección General de Conservación de la Naturaleza). Los autores expresan su agradecimiento.

\section{Referencias}

BELMONTE SERRATO, F. \& ROMERO DÍAZ, M.A. (1996): «Aproximación a las características climáticas en el área de influencia del campo experimental de El Ardal (Murcia). La representatividad de las series climáticas disponibles». Papeles de Geografía, 23-24: $47-61$. 
CAMARASA BELMONTE, M.; BESCOS ATÍN, A. \& SANCHO COMÍNS, J. (1998): Evaluación del riesgo de erosión en relación con la dinámica ocupacional del suelo a partir de sistemas de información geográfica. En: Investigaciones recientes de la geomorfología española. A. Gómez Ortiz \& F. Salvador Franch (Eds.), Barcelona, 579-592.

GARCÍA RUIZ, J.M.; RUIZ FLAÑO, P. \& LASANTA, T. (1996): Soil erosion after farmland abandonment in submediterranean mountains: A general outlook. In J.L. Rubio y A. Calvo (Eds.): Soil degradation and desertification in mediterranean environments. Geoforma Ediciones, Logroño, 165-184.

ICONA (1996): «Red de estaciones experimentales de seguimiento y evaluación de la erosión y la desertización (RESEL), PROYECTO LUCDEME. Dirección General de Conservación de la Naturaleza, Ministerio de Medio Ambiente, Madrid, 70-73.

LÓPEZ-BERMÚDEZ, F.; ROMERO-DÍAZ, A.; MARTÍNEZ-FERNÁNDEZ, J., 1998: Field Studies: El Ardal, Murcia, Spain. In Atlas of Mediterranean Envirnonments in Europe. The Desertification Context. P. Mairota, J.Thornes \& N. Geeson, Eds. J.Wiley and Sons, Ltd. Chichester, England, 114-115.

MARCO MOLINA, J.A. \& MORALES GIL, A. (1995): Terrazas de cultivo abandonadas en el Sureste penisular: Aspectos evolutivos, Investigaciones Geográficas, 13, 81-90.

MARTÍNEZ FERNÁNDEZ, José; MARTÍNEZ FERNÁNDEZ, Julia, LÓPEZ BERMÚDEZ, F. \& ROMERO DÍAZ, A. (1995): «Land Use and soil-vegetation relationships in a Mediterranean ecosystem: El Ardal, Murcia, Spain. Catena, 25, 153-167.

MOLINA GALLART, D. \& NADAL TERSA, J. (1998): Procesos erosivos en campos abandonados del Parque Natural del Cadí-Moixeró, (Pirineos Orientales). En: Investigaciones recientes de la geomorfología española. A. Gómez Ortiz \& F. Salvador Franch (Eds.), Barcelona, 539-546.

MORGAN, R.P.C. (1986): Soil Erosion \& Conservation. Longman. Essex, England, $298 \mathrm{pp}$.

PADILLA BLANCO, A. (1998): Colonización vegetal en campos abandonados de la provincia de Alicante. Publicaciones de la Universidad de Alicante. 365 pp.

ROMERO DÍAZ, A.; BARBERA, G. \& LÓPEZ BERMÚDEZ, F. (1995): Relaciones entre erosión del suelo, precipitación y cubierta vegetal en un medio semiárido del Sureste de la Península Ibérica. Lurralde, 18: 229-243. San Sebastián.

ROMERO DÍAZ, A.; LÓPEZ BERMÚDEZ, F.; BELMONTE SERRATO, F. (1998): «Erosión y escorrentía en el Campo Experimental de «El Ardal» (Murcia). Nueve años de experiencias». Papeles de Geografía, 27: 129-144.

RUIZ FLAÑO, P. (1993): Procesos de erosión en campos abandonados del Pirineo. Ediciones Geoforma, Logroño $191 \mathrm{pp}$.

SÁNCHEZ GÓMEZ, P. (Cood.) (1998): Flora de Murcia. Claves de identificación e iconografía de plantas vasculares. Diego Marín, Murcia, 378 pp. 